\title{
Design and Analysis of Ring Resonator Based Optical Delay Line
}

\author{
Tejaswini B R, Dr.Chandrika T N \\ Siddaganga Institute of Technology, Tumakuru, India \\ Email: tejaswinibr1995@gmail.com, chandrikasit@gmail.com
}

\begin{abstract}
Generation of optical delay line is one of the greatest challenges in the modern photonics. Optical delay lines are important functional elements in optical science and technology. In the areas of optical signal buffering, optical communication systems, phased arrays antenna and photonic signal processing optical delay line plays a very important role. In this paper, we are presenting a novel structure for optical delay line using ring resonator waveguide. A delay line device consists of three ring resonator waveguides and one bus waveguide. Transfer Matrix method is used to analyze the ring resonator which has been designed for single mode. From the designed delay modeling configuration we can achieve a delay of $\sim 500 \mathrm{ps}$.
\end{abstract}

Key words: Optical delay line, Waveguide, Ring resonator.

\section{INTRODUCTION}

An optical delay line has wide range of application in sensors [1], radio frequency photonics, radars, biosensors [2], and in delay line applications. Generally, there are two methods to generate delays for optical waves one is increasing the optical path length and other is slowing down the velocity of light in the waveguide [3] [4]. A delay line is a device which produces a specific desired delay in the transmitted signal. Delay lines are necessary to store the signal in the optical communication, because no memory devices exist to store the signal in the optical communication. To substitute electronic buffering no optical storage technologies are available. Forcing the optical signal to be converted to an electronic format by using switching operators, SONET/SDH [5] [6].Using the ring resonator in series we can achieve a delay by storing the optical signals for a while. In optical telecommunication networks optical delaying is a valuable function and it has important applications like buffering, signal synchronization, time division multiplexing and so forth [7] [8].

In this paper, we are using three ring resonators as shown in figure1; the input wavelengths are given at input port i.e.Ei1, some part of wavelengths will gets coupled to the ring R1 at resonance condition; remaining wavelengths are passes through output/through port i.e. Et1. The coupled wavelengths passes through all three ring and come back to through port at some delay time.

\section{DELAY LINE DEVICE USING RING RESONATOR}

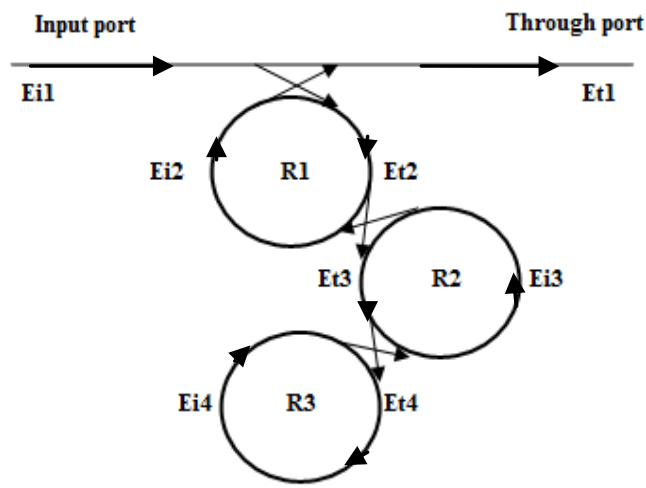

Figure1: Schematic diagram of a delay line device using ring resonator

In the above figure1 shows there are two types of coupling occurs. One is the self coupling i.e. coupling occur inside the bus wave guide or ring resonator waveguide. Another is the cross coupling that occur between the bus waveguide and ring resonator or between two ring resonators waveguide.

\section{THEORETICAL MODELING}

\section{A. Waveguide}

The step index planar waveguide is the simplest optical waveguide structure. The slab waveguide, shown in figure 2 , consist of higher refractive index in the guiding or film region (n1), lower refractive index in substrate region (n2) and cover region (n3).

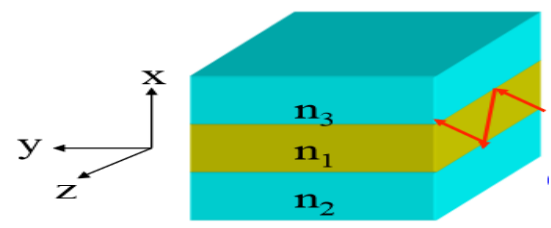

Figure2: Planar (slab) waveguide 
The wave equation for the electric field components in each region can be put in the scalar form

$$
\frac{d^{2} \varphi}{d x^{2}}+\left(k_{0}^{2} n_{i}^{2}-\beta^{2}\right) \varphi=0
$$

Where $n i=n 1, n 2, n 3$ direction.

$\beta$ is a propagation coefficient along $\mathrm{Z}$

The equation inside the core is defined by

$\frac{d^{2} \varphi_{1}}{d x^{2}}+\left(k_{0}^{2} n_{1}^{2}-\beta^{2}\right) \varphi_{1}=0$

Where $k^{2}=k_{0}^{2} n_{1}^{2}-\beta^{2}$ then

$$
\frac{d^{2} \varphi_{1}}{d x^{2}}+k^{2} \varphi_{1}=0
$$

The solution is oscillating function in core region

$$
\varphi(x)=A \sin k x+B \cos k x
$$

The equation of the cladding region is defined by

$$
\frac{d^{2} \varphi_{2}}{d x^{2}}+\left(k_{0}^{2} n_{2}^{2}-\beta^{2}\right) \varphi_{2}=0
$$

Where $\alpha^{2}=-\left(k_{0}^{2} n_{2}^{2}-\beta^{2}\right)$ then

$\frac{d^{2} \varphi_{2}}{d x^{2}}-\alpha^{2} \varphi_{2}=0$

The solution is exponentially decaying in cladding region

$$
\varphi_{2}=C e^{\alpha x}+D e^{-\alpha x}
$$

The characteristic equation is given by

$\tan \left(k \frac{h}{2}\right)=\frac{\gamma}{k}$

Where $k$ is transverse wave number

$\gamma$ is attenuation coefficient $t=$ core thickness

By solving the above characteristic equation using graphical method in MATLAB gives below result.
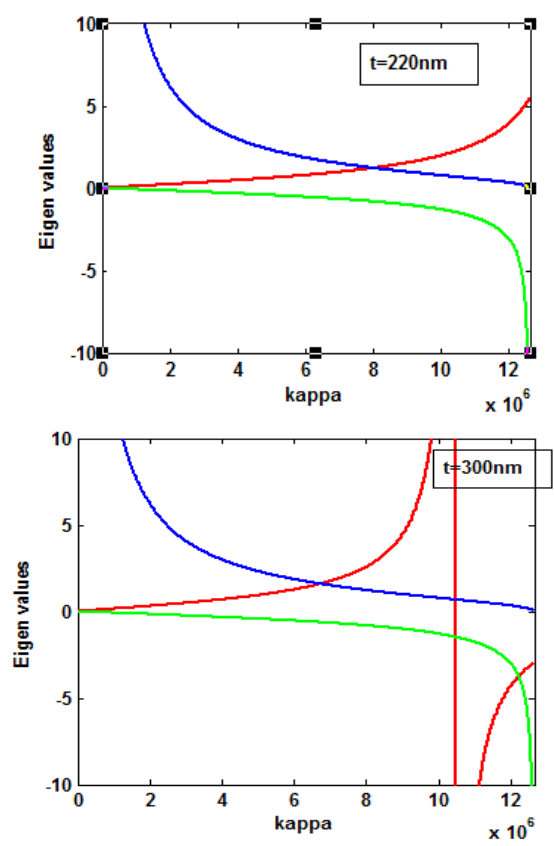

Figure3: Eigen value equation and kappa for TE mode for different value of core thickness
From the above figure 3 we get the different values of kappa foe the different thickness of the core. Here core refractive index (n1) is 3.441 , material or cladding refractive index (n2) is 1.45 and the wavelength $(\lambda)$ at $1550 \mathrm{~nm}$. By substituting the kappa values in below given equation,we can find the propagation constant $\beta$.

$$
\left.\beta=\sqrt{\left(k * n_{1}\right.}\right)^{2}-(k a p p a)^{2}
$$

$k=\frac{2 \pi}{\lambda}$

Where $n_{f}=$ refractive index of the film

From $\beta$ we can calculate the $n_{\text {eff }}$ by using

$$
n_{\text {eff }}=\frac{\beta}{k}
$$

Where $n_{\text {eff }}=$ effective refractive index

\section{B. Ring resonators}

An optical ring resonator is a set of waveguides in which at least one is a closed loop coupled to some sort of light input and output. Ring resonator is a frequency dependent and multifunctional device. Compare to Directional coupler and Mach zehnder interferometer we can use the chip area very effectively in the ring resonator.

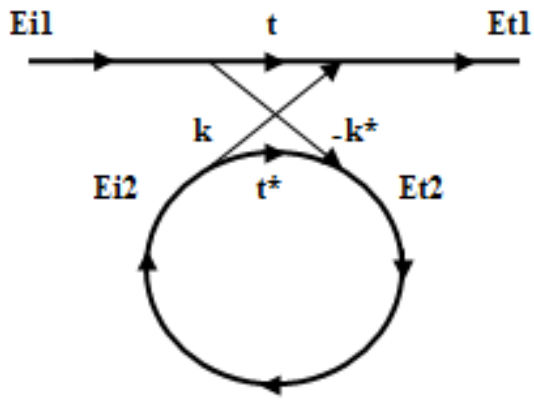

Figure4: Model of a single ring resonator with one waveguide

The input and through port relation in matrix form is

$$
\left(\begin{array}{c}
E_{t 1} \\
E_{t 2}
\end{array}\right)=\left(\begin{array}{cc}
t & k \\
-k^{*} & t^{*}
\end{array}\right)\left(\begin{array}{c}
E_{i 1} \\
E_{i 2}
\end{array}\right)
$$

Where $t$ : self coupling coefficient

$\mathrm{k}$ : cross coupling coefficient.

The $*$ denotes the complex conjugated value of $\mathrm{t}$ and $\mathrm{k}$.

For lossless coupling to occur, the following equation must satisfy.

$$
|k|^{2}+|t|^{2}=1
$$

For the three ring resonators shown in the delay configuration figure1, the input and through port matrix relation is given by

$$
\begin{gathered}
\left(\begin{array}{l}
E_{t 4} \\
E_{i 4}
\end{array}\right)= \\
\left(\begin{array}{cc}
-t_{1} / k_{1} & 1 / k_{1} \\
-1 / k_{1} & t_{1} / k_{1}
\end{array}\right)\left(\begin{array}{cc}
-t_{2} / k_{2} & 1 / k_{2} \\
-1 / k_{2} & t_{2} / k_{2}
\end{array}\right)\left(\begin{array}{cc}
-t_{3} / k_{3} & 1 / k_{3} \\
-1 / k_{3} & t_{3} / k_{3}
\end{array}\right)\left(\begin{array}{l}
E_{i 1} \\
E_{t 1}
\end{array}\right) * \\
\text { X ---- (8) }
\end{gathered}
$$


Where $\mathrm{X}=\alpha 1 e^{\wedge}(j \theta 1) * \alpha 2 e^{\wedge}(j \theta 2) *$ $\alpha 3 e^{\wedge}(j \theta 3)$

\section{SIMULATION AND RESULTS}

Simulation has been carried out using MAT lab and Lumerical. A single ring resonator has been analysed using Lumerical and results are shown in figure 5. In figure 5, through port signal, blue line indicates the unresonated wave signal coming at through port and the green line indicates the resonated wave signal coming at the drop port, which has been coupled into the ring. By using the lumerical simulation tool we have designed for the one ring resonator waveguide and two bus waveguide. We got a delay of $\sim 300$ ps at the drop port. The same is verified using the MATLAB.

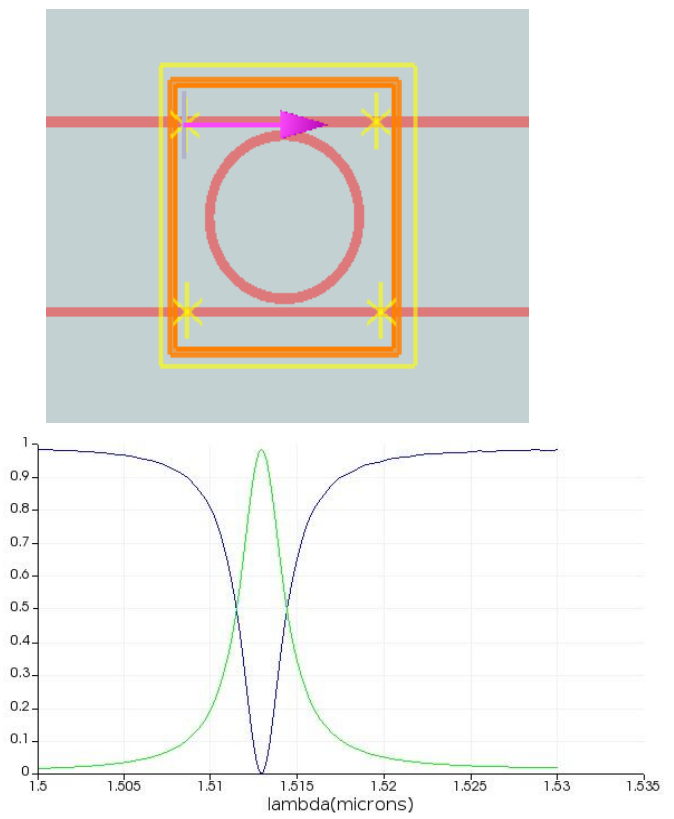

Figure5: Schematic of the ring resonator and spectrum in lumerical tool

MAT lab code is written for equation 8 and got result as shown in figure 6 . Figure 6 is the result of three ring resonators. Though figure 5 and figure 6 have spectrum, there is a difference in delay and attenuation of the signal.

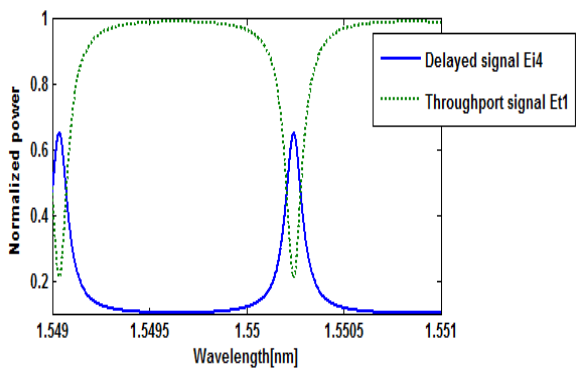

Vol. 2 (12), June 2019, www.ijirase.com
Figure6: Transmission and delayed signal spectrum The analysis of three ring resonators is shown in figure5. Analysis is performed by taking normalized power versus wavelength. Dotted line indicates the unresonated wave signal coming at through port Et1, shown in figure1. And thick line indicates the resonated wave signal coming at Ei4, shown in figure1. From the figure 6 we observed that only certain wavelengths of the signal, coupled into the ring and travels in it. Because of this delay is introduced in the signal.

\section{DELAY CALCULATION}

The delay can be calculated by using the formula given below.

$$
\text { delay }=\frac{1}{F S R}
$$

For approximate value of delay calculation the following equation can be use

$$
F S R=\frac{\lambda^{2}}{n_{g} L} .
$$

WhereFSR: Field spectral range,

$\lambda$ : Wavelength of the signal

$n_{g}$ : group refractive index,

$L=2 \pi r:$ Circumference of ring

We can calculate the radius of ring resonator using

$$
m \lambda_{m}=n_{\text {eff }} * 2 \pi * r
$$

Where $\mathrm{m}=1$ for single mode

Further, we found delay of 500ps for the proposed device.

\section{CONCLUSION}

In conclusion, we have proposed and theoretically analyzed a novel delay line device using ring resonator for communication application. Transfer matrix method has been used to analyze the structure. The device configuration is designed in a way that, the area occupied by it is optimized. A delay of 500ps has been achieved.

\section{ACKNOWLEDGEMENTS}

We thank Prof. T Srinivas, Dept. of ECE, IISc , Bangalore for proving the space to work in his lab.

\section{REFERENCES}

1. "Mathematical modeling of optical MEMS differential pressure sensor using waveguide Bragg gratings embedded in Mach Zehnder interferometer" Chandrika Thondagere1 , Archana Kaushalram1, Talabattula Srinivas1 and Gopalkrishna Hegde2, Published 
10 July 2018, 2018 IOP Publishing Ltd, Journal of Optics, Volume 20, Number 8

2. T. N. Chandrika, H. Dessalegn and T. Srinivas, "Ultra low-cost optical biosensor based on guided mode resonance grating filter," 2015 Workshop on Recent Advances in Photonics (WRAP), Bangalore, 2015, pp. 1-4.

3. X. Wang, L. Zhou, R. Li, J. Xie, L. Lu and J. Chen, "Nanosecond-range continuously tunable silicon optical delay line using ultra-thin silicon waveguides," 2016 Conference on Lasers and Electro-Optics (CLEO), San Jose, CA, 2016, pp. 1-2.

4. D. Melati, A. Waqas, Z. Mushtaq and A. Melloni, "Wideband Integrated Optical Delay Line Based on a Continuously Tunable MachZehnder Interferometer," in IEEE Journal of Selected Topics in Quantum Electronics, vol. 24, no. 1, pp. 1-8, Jan.-Feb. 2018.

5. P. Sethi and S. K. Selvaraja, "CMOSCompatible Silicon Micro-Ring Resonator Based Optical Delay Lines," in 13th International Conference on Fiber Optics and Photonics, OSA Technical Digest (online) (Optical Society of America, 2016), paper Th3A.12.

6. Wang, Junjia et al. "Subwavelength grating enabled on-chip ultra-compact optical true time delay line." Scientific reports (2016).

7. C. Xiang, M. L. Davenport, J. B. Khurgin, P. A. Morton and J. E. Bowers, "Low-Loss Continuously Tunable Optical True Time Delay Based on Si3N4 Ring Resonators," in IEEE Journal of Selected Topics in Quantum Electronics, vol. 24, no. 4, pp. 1-9, July-Aug. 2018

8.S. Ravindran, Y. T. Lee and K. Alameh, "Electroabsorptive double ring resonators for wavelength switching applications," 2009 9th International Conference on Numerical Simulation of Optoelectronic Devices, Gwangju, 2009, pp. 47-48

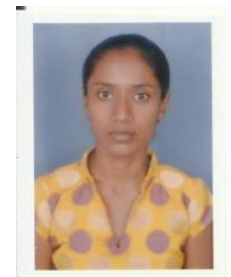

Tejaswini B R was born in tumakuru, India, in 1995.She is a M.Tech student in digital communication branch at Siddaganga Institute of Technology. She received the Bachelor of Engineering in Electronic and Communication Engineering at Shridevi Institute of Engineering and Technology in 2017. During the master's study, she focused on the generation of optical delay lines using the ring resonator. Her current research interest include in delay lines

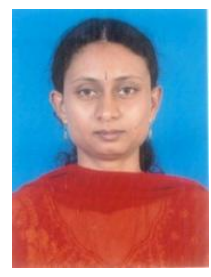

Chandrika T .N. received the M.Tech (Industrial Electronics) from Sri Jayachamarajendra college of Engineering, Mysore, India, in 2007.She pursued her $\mathrm{Ph}$ D. degree from the department of Electrical communication Engineering , Indian Institute of Science (IISc), Bangalore, India. She is currently working as Assistant Professor at Siddaganga Institute of Technology, Tumkur. Her main research interests are in Integrated optics devices, Optical sensors, optical fiber communication 